\title{
The human Transmembrane Protease Serine 2 is necessary for the production of Group 2 influenza A virus pseudotypes
}

\author{
Francesca Ferrara ${ }^{\dagger}$, Eleonora Molesti ${ }^{\dagger}$, Eva Böttcher-Friebertshäuser ${ }^{\ddagger}$, Giovanni Cattoli ${ }^{\ddagger}$, Davide \\ Corti $^{\psi}$, Simon D Scott ${ }^{\dagger *}$ and Nigel J Temperton ${ }^{\dagger *}$
}

†Viral Pseudotype Unit, Medway School of Pharmacy, University of Kent, Central Avenue, Chatham Maritime, ME4 4TB, UK, Institute of Virology, Philipps University Marburg, Hans-Meerwein-Str. 2, 35043 Marburg, Germany, ${ }^{\sharp}$ FAO-OIE and National Reference Laboratory for Newcastle Disease and Avian Influenza, Istituto Zooprofilattico delle Venezie, Viale dell'Università, 1035020 Legnaro (PD), Italy, ${ }^{\top}$ Institute for Research in Biomedicine, Via Vela 6, 6500 Bellinzona, Switzerland.

*Correspondence to: Simon Scott, Email: s.d.scott@kent.ac.uk, or Nigel Temperton, Email: n.temperton@kent.ac.uk, Tel: +44 (0)1634 202957, Fax: +44 (0)1634 883927

Received: 31 October 2012; Revised: 19 February 2013; Accepted: 20 February 2013; Published: 20 February 2013

(C) Copyright The Author(s). First Published by Library Publishing Media. This is an open access article, published under the terms of the Creative Commons Attribution Non-Commercial License (http://creativecommons.org/licenses/ by-nc/2.5). This license permits non-commercial use, distribution and reproduction of the article, provided the original work is appropriately acknowledged with correct citation details.

\begin{abstract}
The monomer of influenza haemagglutinin is synthesized as a single polypeptide precursor that during maturation is cleaved by proteases into two active subunits. Other studies have demonstrated that the human Transmembrane Protease Serine 2 (TMPRSS2) can cleave the HA of human seasonal influenza viruses. Consequently, we have investigated the use of human Transmembrane Protease Serine 2 to produce high titre influenza haemmagglutinin (HA) lentiviral pseudotypes from Group 2 influenza viruses. Such pseudotypes represent powerful and safe tools to study viral entry and immune responses. Influenza pseudotype particles are obtained by co-transfecting human embryonic kidney HEK293T/17 cells using plasmids coding for the influenza HA, HIV gag-pol and a lentiviral vector incorporating firefly luciferase. However, in order to produce Group 2 pseudotypes, it was necessary to co-transfect a plasmid expressing the TMPRSS2 endoprotease, to achieve the necessary HA cleavage for infective particle generation. These lentiviral pseudotypes were shown to transduce HEK293T/17 cells with high efficiency. This demonstrates that TMPRSS2 is necessary for the functional activation, in vitro, of both the HA of human seasonal influenza and other Group 2 HA influenza strains. Additionally, we show that the Group 2 influenza pseudotype particles can be used as surrogate antigens in neutralization assays and are efficiently neutralized by corresponding influenza virus reference sera. These data demonstrate that the viral pseudotype system is a powerful method for serological surveillance of a wide range of influenza viruses.
\end{abstract}

KEYWORDS: Haemagglutinin, protease cleavage, antibody response, pseudotype serology

\section{INTRODUCTION}

Influenza A viruses are disease-causing agents in humans and animals, which have a reservoir in wild birds, especially Charadriiformes and Anseriformes (Olsen et al, 2006). Influenza A viruses are classified using the antigenic characteristic of the envelope glycoproteins haemagglutinin (HA) and neuraminidase (NA). Currently, using antigenic characteristics and phylogenetic analysis, 17 different haemagglutinin subtypes are recognized and are divided into two lineages: Group-1 and Group-2 (Figure 1) (Röhm et al, 1996; Fouchier et al, 2005; Tong et al, 2012). Of the 17 haemagglutinin subtypes only two, $\mathrm{H} 1$ and $\mathrm{H} 3$, cause seasonal epidemics in the human population. However, viruses belonging to other subtypes have shown the capacity for avian-to-human transmission and their spread needs to be monitored in avian and mammalian populations for pandemic preparedness (Peiris, 2009). This can be achieved using serological and molecular biological diagnostic methods that permit the identification of haemagglutinin subtypes 


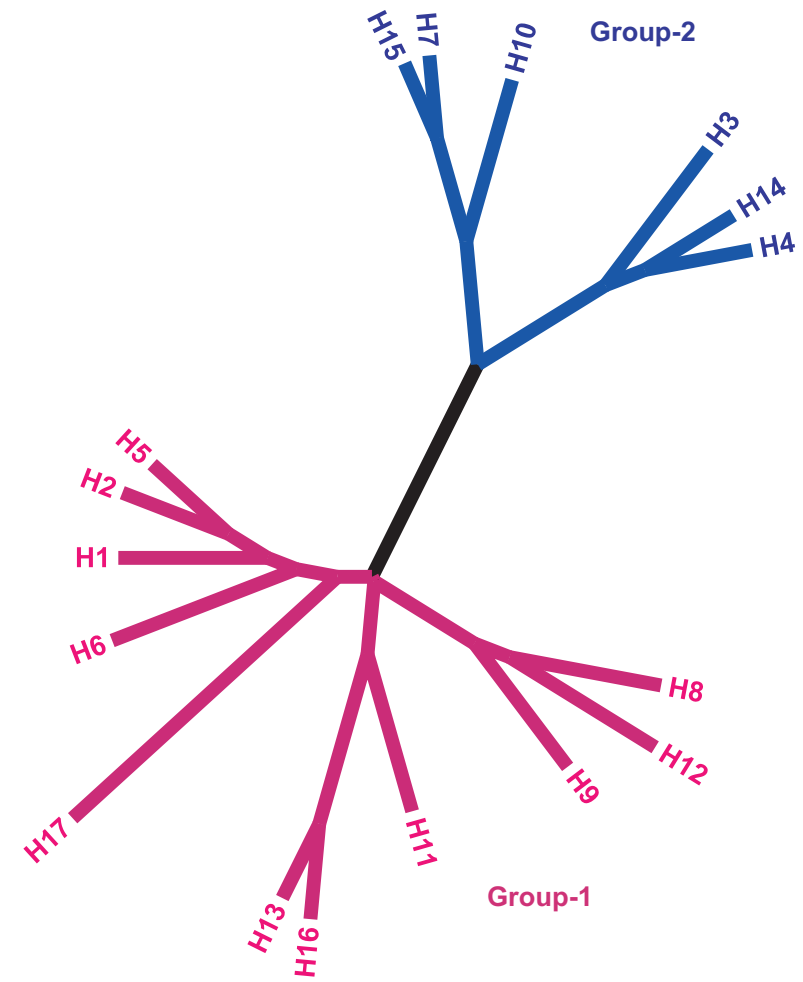

Figure 1. Phylogenetic relationship of influenza A haemagglutinin subtypes. Evolutionary analysis involving 17 amino acid sequences representing HAs from each subtype was conducted in MEGA5 (Tamura et al, 2011). The evolutionary history was inferred using the Neighbor-Joining method, computing the evolutionary distances utilizing the Dayhoff matrix-based method. All positions with less than $100 \%$ site coverage were eliminated. Colours were added with FigTree software (http://tree.bio.ed.ac.uk/).

(Cattoli and Terregino, 2008). The haemagglutinin is the major target of antibody response against influenza viruses. It is the trimeric envelope glycoprotein necessary for entry into susceptible cells. Each HA monomer is produced as a single polypeptide precursor, HA0, that during maturation is cleaved into two subunits, HA1 and HA2. This process is a necessary step to acquire fusion activity and infectivity of influenza. When multiple basic amino acid residues (lysine and arginine) are situated immediately before the cleavage arginine, the haemagglutinin is cleaved by ubiquitously expressed subtilisin-like endoproteases, such as furin. This determines the virulence and lethality for several avian species observed in the high pathogenicity avian influenza (HPAI) strains H5 and H7 (Garten and Klenk, 2008). On the other hand, cleavage at the single arginine (or lysine) amino acid at the HA cleavage site of low pathogenicity avian influenza (LPAI) strains, human and mammalian influenza viruses is thought to be mediated by tissue-specific proteases. Recently, different human lung proteases belonging to the serine protease family that are able to cleave the haemagglutinin of human influenza strains were identified: Transmembrane Protease Serine 2 and 4 (TMPRSS2/4) and Human Airway Trypsin-like protease (HAT) (Böttcher et al, 2006; Bertram et al, 2010). To date, the proteases that cleave the haemagglutinin of LPAI strains are still unidentified. To study the HA cleavage of human seasonal strains (H1 and H3) by TMPRSS2, HAT and their avian and mammalian homologous proteases, pseudotype lentiviral particles are a suitable approach (Bertram et al, 2012). Influenza pseudotype particles (pp) represent powerful and safe tools to study both the antibody response directed against the influenza haemagglutinin and the viral entry mechanism (Garcia and Lai, 2011). In the present study, we use a pseudotype generation system to investigate human TMPRSS2 and its role in mediating the conversion of human and LPAI HA0s into their active forms, and we show that the pseudotypes produced can be efficiently used in a virus neutralization assay.

\section{MATERIALS AND METHODS}

\section{Haemagglutinin expressing plasmids}

The haemagglutinin of A/Udorn/307/1972 (H3N2) and of A/chicken/Italy/1082/1999 (H7N1) were cloned in the expression plasmid pI.18 using methodology described elsewhere (Temperton et al, 2007). The haemagglutinin of A/ duck/Czechoslovakia/1956 (H4N6), A/chicken/Germany/ N49 (H10N7), A/mallard/Astrakhan/263/1982 (H14N5), A/shearwater/West Australia/2576/1979 (H15N9) were custom synthesised and codon-optimized (Genscript), and cloned into the phCMV1 vector (Genlantis) as previously reported (Corti et al, 2011).

\section{Reference sera}

Reference sheep serum against A/England/427/1988 (H3N2) was provided by the National Institute for Biological Standards and Controls (NIBSC, United Kingdom). World Organisation for Animal Health (OIE) avian reference hyperimmune sera against H3N8, H4N8, H14N5, H10N1 and $\mathrm{H} 15 \mathrm{~N} 9$ were produced in specific pathogen-free chickens as described elsewhere (Cattoli et al, 2011). Reference avian sera against A/African starling/England/983/1979 (H7N1), A/chicken/Wales/1306/2007 (H7N2), A/chicken/ England/4054/2006 (H7N3), A/England/268/1996 (H7N7) were provided by the Animal Health and Veterinary Laboratories Agency (AHVLA).

\section{Cell line}

Human embryonic kidney HEK293T/17 cells were cultured in Dulbecco's Modified Eagle Medium (DMEM) with Glutamax and high glucose (Gibco, Invitrogen, UK), supplemented with 10\% (v/v) fetal bovine serum (Sigma) and 1\% (v/v) penicillin/streptomycin (Fisher Bioreagents).

\section{Pseudotype lentiviral particles production and titration}

Lentiviral pseudotyped particles were produced as described previously (Temperton et al, 2007; Corti et al, 2011). Briefly, the HIV gag-pol plasmid p8.91, the firefly luciferase expressing plasmid pCSFLW, the HA expressing plasmid and the pCAGGS-TMPRSS2 (Böttcher et al, 2006) were co-transfected into human embryonic kidney HEK293T/17 cells using Fugene-6 transfection reagent (Roche, UK). After 24 hours incubation, $1 \mathrm{U}$ of recombinant neuraminidase from Clostridium perfringens (Sigma) was added (Figure 2) to facilitate pseudotype exit from the producer cells. Fortyeight hours post-transfection supernatant was harvested, filtered through $0.45 \mu \mathrm{m}$ filters and stored at $-80^{\circ} \mathrm{C}$. Pseudotyped particles were also produced in the absence of pCAGGS-TMPRSS2 plasmid ( $\triangle$ TMPRSS2). Titration of the pseudotype particles was performed by transducing HEK293T/17 cells $\left(1 \times 10^{4}\right.$ cells/well $)$, with $50 \mu$ of neat pseudotype supernatant and subsequent 1:2 serial dilutions. 


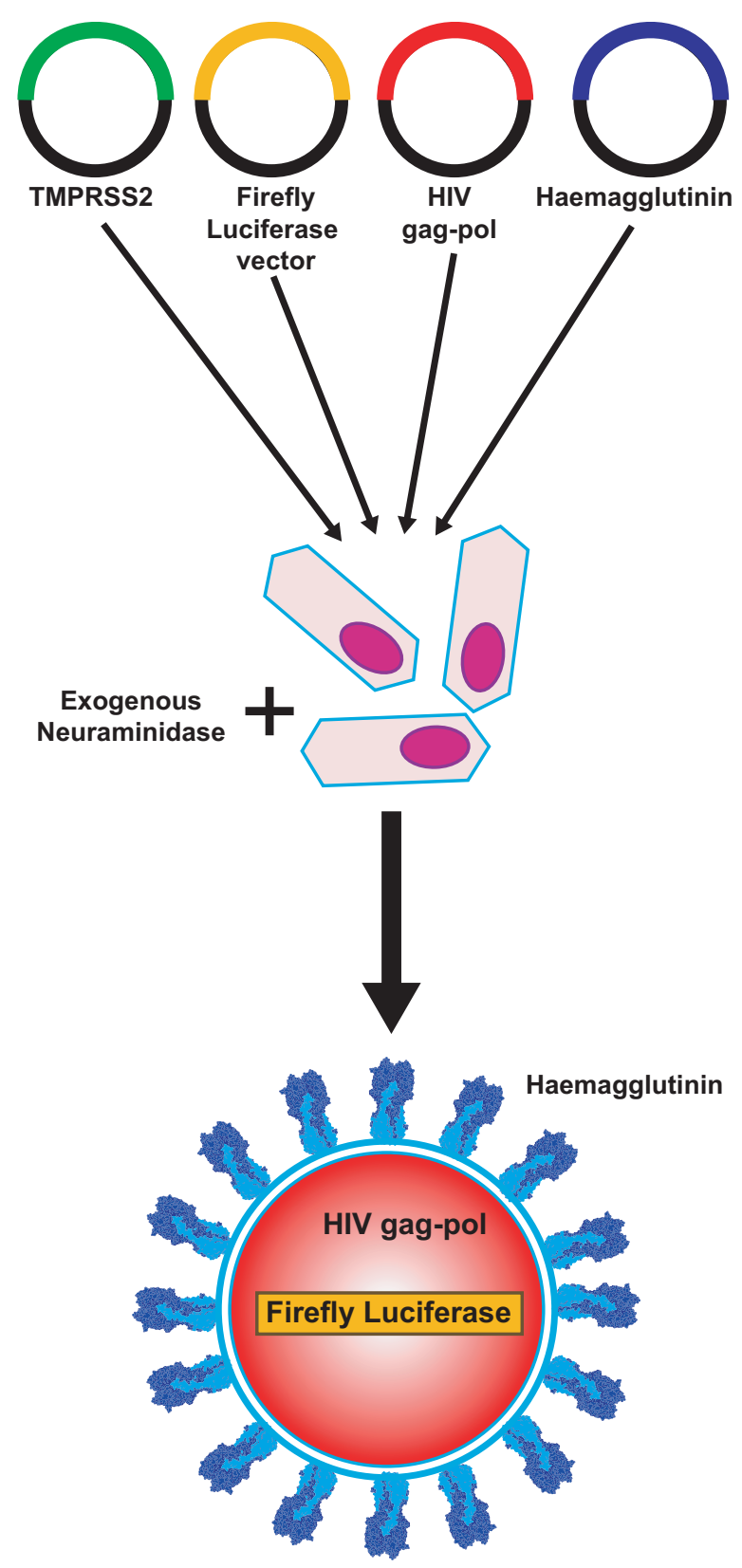

Figure 2. Schematic representation of Group-2 influenza pseudotype production. Plasmids encoding HIV gag-pol, the specific HA, TMPRSS2 protease and a Firefly Luciferase encoding lentiviral vector are transfected into HEK293T/17 cells. $24 \mathrm{hr}$ post-transfection, exogenous neuraminidase is added. $48 \mathrm{hr}$ post-transfection, influenza pseudotypes are harvested.

The $\triangle$ TMPRSS2 influenza pseudotypes were titrated in the absence or presence of TPCK-Trypsin to evaluate the effect of endogenous TMPRSS2 expression and to activate the haemagglutinin to obtain functional pseudotypes. TPCK-Trypsin (Sigma) was added to the pseudotypes to obtain the final concentration of $50 \mu \mathrm{g} / \mathrm{ml}$ and maintaining constant the TPCK-Trypsin concentration during the serial dilution. After $30 \mathrm{~min}$ at $37^{\circ} \mathrm{C}, 50 \mu \mathrm{l}$ of trypsin neutralizing solution (Lonza) were added to each well. After $48 \mathrm{hrs}$, luminescence of cell cultures (in relative luminescence units or RLUs) was evaluated by luminometry using the Bright-Glo assay system (Promega, UK). Titres were expressed in RLU/ml.

\section{Pseudotype particle neutralization assay}

\section{with reference sera}

Reference sera were tested against their respective pseudotypes by serially diluting $1: 2$ in culture media (from $1: 80$ to 1:5120) in white 96-well flat-bottomed tissue culture plates (Nunc) and $50 \mu 1$ of pseudotype supernatant were added in each well, diluted to have a final concentration of $1 \times 10^{6}$ RLU/well. A 'cell only' control and a 'pseudotype only' (no sera) control were used in each assay. After $1 \mathrm{hr}$ incubation of sera plus virus at $37^{\circ} \mathrm{C}, 1 \times 10^{4} \mathrm{HEK} 293 \mathrm{~T} / 17$ cells were added to each well and the plates were incubated for a further $48 \mathrm{hrs}$ at $37^{\circ} \mathrm{C}$. Cell luminescence was measured as described above. The results were normalized and expressed as the percentage of inhibition of pseudotype virus entry (neutralization) using the cell only and pseudotype only control RLU values. The half maximal inhibitory concentration $\left(\mathrm{IC}_{50}\right)$ and the $90 \%$ inhibitory concentration $\left(\mathrm{IC}_{90}\right)$ were calculated with Prism version 6 (GraphPad Software).

\section{RESULTS}

The titres of Group-2 influenza pseudotype particles expressed in RLU/ml are shown in Figure 3. Through cotransfection with pCAGGS-TMPRSS2, we successfully obtained high-titre viral pseudotype particles harbouring the haemagglutinin of a human H3N2 virus(A/Udorn/307/1972), A/duck/Czechoslovakia/1956 (H4N6), A/chicken/Germany/ N49 (H10N7), A/mallard/Astrakhan/263/1982 (H14N5), A/shearwater/West Australia/2576/1979 (H15N9) and H7 LPAI virus A/chicken/Italy/1082/1999 (H7N1). Notably, $\mathrm{H} 3, \mathrm{H} 4$ and $\mathrm{H} 10$ pseudotypes were produced with titres above $10^{9} \mathrm{RLU} / \mathrm{mL}$. The pseudotype particle titres obtained with TMPRSS2 transfection were compared to the controls: $\triangle$ TMPRSS2 influenza pseudotypes, $\triangle$ TMPRSS2 TPCK-Trypsin activated influenza pseudotypes and pseudotype particles produced without the HA envelope protein $(\triangle$ envelope). These data indicate that TMPRSS2 activates the haemagglutinin not only of human strains, such as A/ Udorn/307/1972 (H3N2), but also the haemagglutinin of avian influenza strains (H4, H14, H15, H7 LPAI and H10 subtypes) in a similar manner to TPCK-Trypsin.

When tested in neutralization assays, the pseudotype particles were efficiently neutralized by their paired antisera (Table 1) using an $\mathrm{IC}_{50}$ cut-off dilution of 1:80. The only exception was for A/Udorn/307/1972 (H3N2) pseudotype that is efficiently neutralized $\left(\mathrm{IC}_{50}>1: 5120\right)$ by the antiA/England/427/1988 (H3N2) sheep serum, but moderately neutralized by the anti-H3N8 sera of avian origin $\left(\mathrm{IC}_{50}=1: 160-1: 320\right)$. Different neutralization profiles were also observed between the different $\mathrm{H} 7$ antisera, with the anti-A/chicken/England/4054/2006 (H7N3) exhibiting the least potent neutralization activity $\left(\mathrm{IC}_{50}=1: 1280-1: 2560\right)$.

\section{DISCUSSION}

We have shown the essential role of TMPRSS2 in the production of high-titre Group-2 influenza pseudotype particles: the protease co-expression during virus production mediates the haemagglutinin activation of Group-2 human influenza and LPAI virus strains and enables functional pseudotype particles that have titres comparable to or higher than the ones that can be achieved with TPCK-Trypsin treatment. 


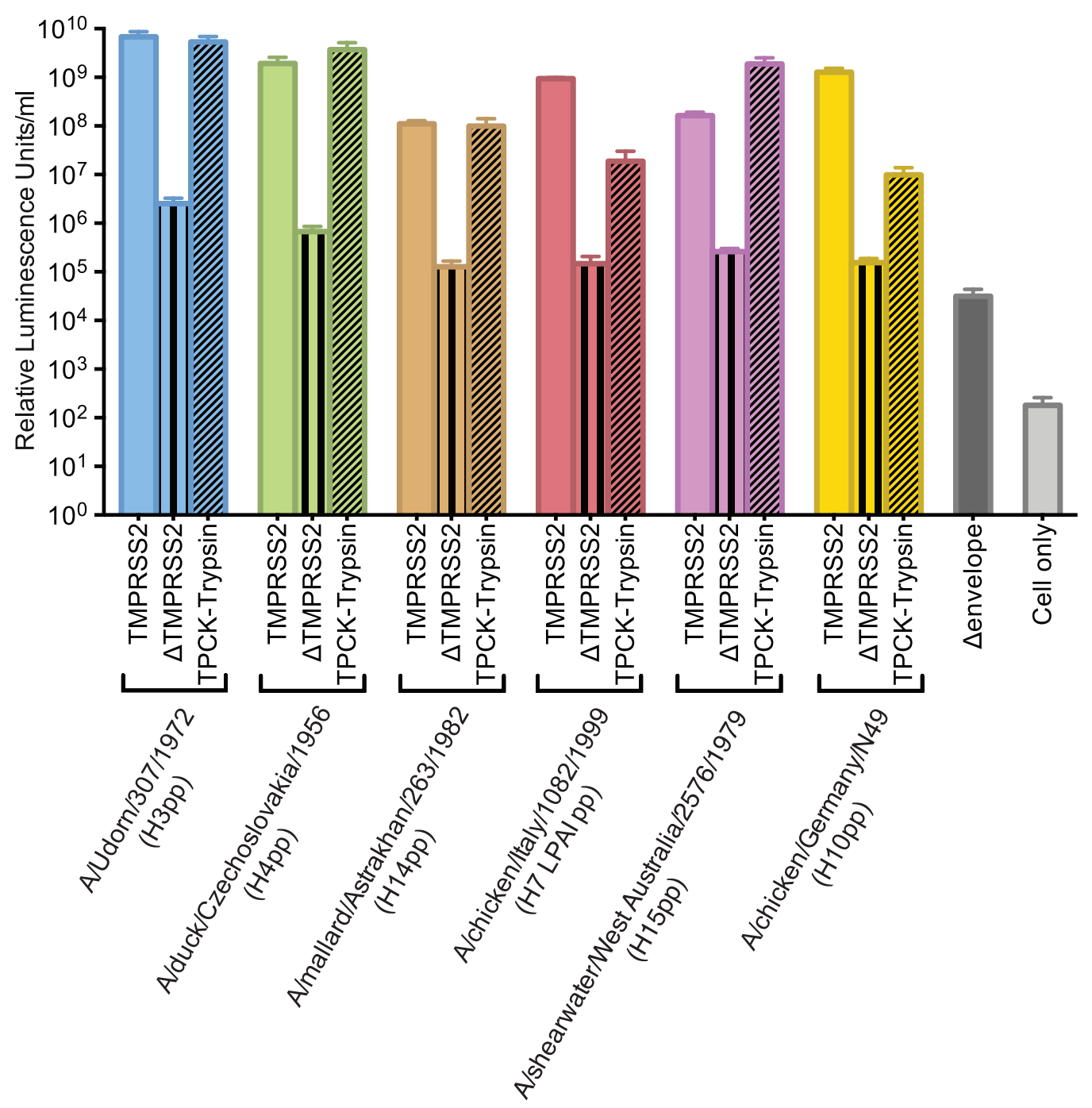

Figure 3. Titres of Group-2 influenza pseudotype particles. Pseudotype transduction titres expressed in Relative Luminescence Units per $\mathrm{ml}( \pm \mathrm{SEM})$ are shown. The titres of influenza pseudotype particles produced after transfection of the pCAGGS-TMPRSS2 plasmid and the titre of $\triangle$ TMPRSS2 influenza pseudotype particles, before and after the TPCK-trypsin treatment, are reported. $\Delta$ envelope (pseudotype produced without the HA envelope protein) and cell only controls are also included.

Since TMPRSS2 is a protease that is able to cleave human influenza haemagglutinins (Böttcher et al, 2006) and TPCKTrypsin is able to activate haemagglutinin by proteolitic cleavage, we can assume that TMPRSS2 activates the HA on pseudotype particles by specific cleavage.

The fact that human TMPRSS2 is able to activate, most probably by cleavage, the HAs of different influenza A subtypes reinforces the concept that haemagglutinin cleavage is a conserved process between avian species and humans, which was highlighted by the observation that TMPRSS2 homologous proteins are able to cleave human influenza HA (Bertram et al, 2012). However further studies need to be performed to determine the function of TMPRSS2 and other proteases (e.g., HAT) in haemagglutinin activation and cleavage, to examine the cleavage kinetics and to understand the haemagglutinin amino acid residues involved in the haemagglutinin-protease interaction. Furthermore, it will be interesting to evaluate the ability of TMPRSS2 to activate LPAI Group-1 influenza haemagglutinin subtypes, since this protease is able to cleave the human $\mathrm{H} 1$ subtype (Böttcher et al, 2006) and similarly produced H1 pseudotypes have been reported in the literature (Wang et al, 2008; Corti et al, 2011). In the present study, we show that although human TMPRSS2 activates the haemagglutinin of different Group-2 subtypes, it is not clear if the difference in the titres observed between subtypes are dependent on differential HA expression and maturation (glycosylation, endoplasmic reticulum and Golgi apparatus trafficking) in the HEK293T/17 producer cell line (Ueda et al, 2008) or on differential TMPRSS2 recognition and cleavage of the different subtype haemagglutinins.

The Group-2 influenza haemagglutinins used in this study are characterized by the cleavage motif $P[E, G][K, V, I]$ $\mathrm{XX}[\mathrm{K}, \mathrm{R}]$, with the numbers and physicochemical properties of the central aminoacidic residues between the proline and the cleavage arginine/lysine variable between subtypes (H3: PEKQTR; H4: PEKASR; H14: PGKQAK; H7: PEIPKGR; H15: PEKIRTR; H10: PEVVQGR). Recently the structure of an H16 subtype haemagglutinin was resolved and it was observed that some amino acid residues upstream of the 
Table 1. Half maximal inhibitory concentration $\left(\mathrm{IC}_{50}\right)$ and the $90 \%$ inhibitory concentration $\left(\mathrm{IC}_{90}\right)$ of reference antisera against Group-2 influenza pseudotype particles (pp). $\mathrm{IC}_{50}$ and $\mathrm{IC}_{90}$ were calculated with Graph Pad Prism version 6 and then were expressed as assay dilutions.

\begin{tabular}{|l|l|l|l|}
\hline & & $\mathbf{I C}_{\mathbf{9 0}}$ & $\mathbf{I C}_{\mathbf{5 0}}$ \\
\hline \multirow{2}{*}{ H3pp } & anti-H3N2 & $1: 1280$ & $>1: 5120$ \\
\cline { 2 - 4 } & anti-H3N8 & $<1: 40$ & $1: 160-1: 320$ \\
\hline \multirow{2}{*}{ H4pp } & anti-H4N8 & $1: 2560-1: 5120$ & $>1: 5120$ \\
\hline \multirow{2}{*}{ H14pp } & anti-H14N5 & $>5120$ & $>1: 5120$ \\
\hline \multirow{2}{*}{$\begin{array}{l}\text { H7pp } \\
\text { (LPAI) }\end{array}$} & anti-H7N1 & $>1: 5120$ & $>1: 5120$ \\
\cline { 2 - 4 } & anti-H7N2 & $1: 2560-1: 5120$ & $>1: 5120$ \\
\cline { 2 - 4 } & anti-H7N7 & $1: 320-1: 640$ & $1: 1280-1: 2560$ \\
\hline \multirow{2}{*}{ H15pp } & anti-H15N9 & $>1: 5120$ & $>1: 5120$ \\
\hline \multirow{2}{*}{ H10pp } & anti-H10N1 & $1: 2560-1: 5120$ & $>1: 5120$ \\
\hline
\end{tabular}

cleavage site are important in determining the surface accessibility of the cleavage arginine (Lu et al, 2012). A similar process could explain the slightly lower titres observed in H14 and H15 pseudotypes: H14 haemagglutinin subtypes are characterized by a single lysine in their cleavage site (PGKQAK) (Kawaoka et al, 1990) and in H15 some amino acid residues upstream of the cleavage site (PEKIRTR) differ from the closely related H7 subtypes (PEIPKGR) (Röhm et al, 1996).

The resolving of the human TMPRSS2 protein structure and co-crystallization of TMPRSS2 with human and/or avian haemagglutinin could be valuable to delineate the amino acid residues involved in the cleavage process. This would not only enhance understanding of the haemagglutinin activation mechanism, but importantly may aid development of potential protease inhibitors (Zhirnov et al, 2011; Böttcher-Friebertshäuser et al, 2012), in a similar manner to neuraminidase inhibitor design, for treatment of severe influenza virus infections. The panel of Group-2 influenza pseudotypes described in this paper, in addition to those previously produced, could be utilized as tools to test the activity of these compounds in a pre-clinical screening phase.

The Group-2 influenza pseudotype particles could also be used to study the antibody response directed against haemagglutinin. Serological methods, such as haemagglutination inhibition and microneutralization, are cost-effective and widely-used methodologies to monitor the circulation and the prevalence of influenza viruses and are also employed in vaccine efficacy studies (Cattoli and Terregino, 2008). In this report, we have demonstrated that reference influenza antisera are able to efficiently neutralize their haemagglutinin-paired influenza pseudotype particles. It has been previously demonstrated that influenza pseudotype particle neutralization assays correlate with other classical serological assays (Temperton et al, 2007; Alberini et al,
2009; Garcia et al, 2010) and may become the technique of choice for HPAI H5 and H7 virus serology. Additionally, since the pseudotypes described are replication-defective, they offer a safe alternative to wild type virus methods that require biosafety level 3 containment, and the detection of antibody response is not influenced by the variability of blood-based reagents as observed in other assays (i.e. erythrocytes in haemagglutination inhibition) (Rowe et al, 1999). Furthermore, the pseudotype neutralization assay appears to be more effective than other functional assays to detect the antibody response directed against the HA2 haemagglutinin stalk (Corti et al, 2011). This is most likely due to the fact that, with the absence of neuraminidase on the pseudotype particle membrane surface, glycoprotein density is reduced and the HA stalk thus becomes more accessible to antibodies. Consequently, pseudotypes could be used to effectively study heterosubtypic antibody responses directed against the HA stalk region. In this study, with the production of Group-2 influenza pseudotypes we present a proof-of-principle for their use as surrogate antigens in antibody neutralization assays. We demonstrate that reference influenza antisera efficiently neutralize the matched pseudotypes. Interestingly, the human A/Udorn/307/1972 (H3N2) pseudotype is not efficiently neutralized by its paired (H3N8) avian antisera compared with the sheep H3N2 antisera. This is probably due to the fact that $\mathrm{H} 3$ avian viruses belong to a different lineage with respect to the human H3 (Zhang et al, 2007). Variation in the antisera neutralizing titres is also observed when the $\mathrm{H} 7$ antisera are tested. This is related to the origin of the antisera: they were generated against different $\mathrm{H} 7$ virus strains from the $\mathrm{H} 7$ pseudotype strain. This also may reflect intra-subtype antigenic differences among H7 strains. Furthermore the different antisera preparations probably have different neutralizing titres against their own viral strain.

Overall, the results presented here reinforce the greater sensitivity of the pseudotype particle neutralization assays described previously (Temperton et al, 2007; Garcia et al, 2010). These neutralization assays using Group-2 influenza pseudotype particles, provide additional impetus to optimise, standardise and validate this technology as a method to be used for the surveillance of influenza viruses in animals and humans.

\section{CONCLUSIONS}

In the present study we have shown that:

- TMPRSS2 functions in effecting the activation, presumably by cleavage, of Group-2 influenza A virus haemagglutinins to produce high-titre influenza pseudotypes.

- Influenza pseudotypes can be employed to determine which proteases are involved in haemagglutinin activation of individual subtypes.

- Group-2 influenza pseudotype particles are neutralized by matched or related reference antisera.

- The Group-2 influenza pseudotype particle neutralization assay could be used as an efficient serological surveillance assay, and for studying the antibody response against haemagglutinin elicited by vaccination or natural infection. 


\section{ACKNOWLEDGEMENTS}

We thank the Animal Health and Veterinary Laboratories Agency for the reference antisera provided. This work was funded by the University of Kent.

\section{COMPETING INTERESTS}

None declared.

\section{LIST OF ABBREVIATIONS}

AHVLA; Animal Health and Veterinary Laboratories Agency HA; Haemagglutinin

HAT; Human Airway-Trypsin-like protease

HPAI; High pathogenicity avian influenza

$\mathrm{IC}_{50} ;$ Half maximal inhibitory concentration

$\mathrm{IC}_{90} ; 90 \%$ inhibitory concentration

LPAI; Low pathogenicity avian influenza

NA; Neuraminidase

NIBSC; National Institute for Biological Standards and Controls

OIE; World Organisation for Animal Health

Pp; Pseudotype particles

RLU; Relative luminescence unit

TMPRSS2; Transmembrane Protease Serine 2

\section{REFERENCES}

Alberini I, Del Tordello E, Fasolo A et al. 2009. Pseudoparticle neutralization is a reliable assay to measure immunity and crossreactivity to H5N1 influenza viruses. Vaccine, 27, 5998-6003.

Bertram S, Glowacka I, Blazejewska P et al. 2010. TMPRSS2 and TMPRSS4 facilitate trypsin-independent spread of influenza virus in Caco-2 cells. J Virol, 84, 10016-10025.

Bertram S, Heurich A, Lavender $\mathrm{H}$ et al. 2012. Influenza and SARS-coronavirus activating proteases TMPRSS2 and HAT are expressed at multiple sites in human respiratory and gastrointestinal tracts. PLoS One, 7, e35876.

Böttcher E, Matrosovich T, Beyerle M et al. 2006. Proteolytic activation of influenza viruses by serine proteases TMPRSS2 and HAT from human airway epithelium. J Virol, 80, 9896-9898.

Böttcher-Friebertshäuser E, Lu Y, Meyer D et al. 2012. Hemagglutinin activating host cell proteases provide promising drug targets for the treatment of influenza A and B virus infections. Vaccine, 30, 7374-7380.

Cattoli G, Milani A, Temperton N et al. 2011. Antigenic drift in $\mathrm{H} 5 \mathrm{~N} 1$ avian influenza virus in poultry is driven by mutations in major antigenic sites of the hemagglutinin molecule analogous to those for human influenza virus. J Virol, 85, 8718-8724.

Cattoli G and Terregino C. 2008. New perspectives in avian influenza diagnosis. Zoonoses Public Health, 55, 24-28.
Corti D, Voss J, Gamblin SJ et al. 2011. A neutralizing antibody selected from plasma cells that binds to group 1 and group 2 influenza A hemagglutinins. Science, 333, 850-856.

Fouchier RA, Munster V, Wallensten A et al. 2005. Characterization of a novel influenza A virus hemagglutinin subtype (H16) obtained from black-headed gulls. J Virol, 79, 2814-2822.

Garcia JM, Lagarde N, Ma ES et al. 2010. Optimization and evaluation of an influenza A (H5) pseudotyped lentiviral particlebased serological assay. J Clin Virol, 47, 29-33.

Garcia JM and Lai JC. 2011. Production of influenza pseudotyped lentiviral particles and their use in influenza research and diagnosis: an update. Expert Rev Anti Infect Ther, 9, 443-455.

Garten W and Klenk H. 2008. Cleavage Activation of the Influenza Virus Hemagglutinin and Its Role in Pathogenesis. In: Klenk H, Garten W and Matrosovich M (Eds) Avian Influenza, Karger, Basel, Switzerland, pp 156-167.

Kawaoka Y, Yamnikova S, Chambers TM et al. 1990. Molecular characterization of a new hemagglutinin, subtype H14, of influenza A virus. Virology, 179, 759-767.

$\mathrm{Lu}$ X, Shi Y, Gao F et al. 2012. Insights into avian influenza virus pathogenicity: the hemagglutinin precursor HA0 of H16 subtype has an $\alpha$-helix structure in its cleavage site with an inefficient HA1/HA2 cleavage. J Virol, 86, 12861-12870.

Olsen B, Munster VJ, Wallensten A et al. 2006. Global patterns of influenza a virus in wild birds. Science, 312, 384-388.

Peiris JSM. 2009. Avian influenza viruses in humans. Rev Sci Tech, 28, 161-173.

Röhm C, Zhou N, Süss J et al. 1996. Characterization of a novel influenza hemagglutinin, H15: criteria for determination of influenza A subtypes. Virology, 217, 508-516.

Rowe T, Abernathy RA, Hu-Primmer J et al. 1999. Detection of antibody to avian influenza A (H5N1) virus in human serum by using a combination of serologic assays. J Clin Microbiol, 37, 937-943.

Tamura K, Peterson D, Peterson N et al. 2011. MEGA5: molecular evolutionary genetics analysis using maximum likelihood, evolutionary distance, and maximum parsimony methods. Mol Biol Evol, 28, 2731-2739.

Temperton NJ, Hoschler K, Major D et al. 2007. A sensitive retroviral pseudotype assay for influenza H5N1-neutralizing antibodies. Influenza Other Respi Viruses, 1, 105-112.

Tong S, Li Y, Rivailler P et al. 2012. A distinct lineage of influenza A virus from bats. Proc Natl Acad Sci USA, 109, 4269-4274.

Ueda M, Yamate M, Du A et al. 2008. Maturation efficiency of viral glycoproteins in the ER impacts the production of influenza A virus. Virus Res, 136, 91-97.

Wang W, Butler EN, Veguilla V et al. 2008. Establishment of retroviral pseudotypes with influenza hemagglutinins from $\mathrm{H} 1$, $\mathrm{H} 3$, and H5 subtypes for sensitive and specific detection of neutralizing antibodies. J Virol Methods, 153, 111-119.

Zhang W, Jiang Q and Chen Y. 2007. Evolution and variation of the $\mathrm{H} 3$ gene of influenza A virus and interaction among hosts. Intervirology, 50, 287-295.

Zhirnov OP, Klenk HD and Wright PF. 2011. Aprotinin and similar protease inhibitors as drugs against influenza. Antiviral Res, 92, 27-36. 